\section{Developmental age and the critical period for imprinting}

\author{
JOHN T. WILLIAMS, JR. \\ University of South Carolina, Columbia, S.C. 29208
}

Developmental age is widely accepted as a more sensitive baseline than posthatch age for the determination of the critical period for imprinting. The original data upon which this hypothesis is based were reexamined and found to have been analyzed in a meaningless way. There seems to be no objective data supporting the idea that developmental age provides a more sensitive baseline.
While summarizing an experiment regarding the critical period for imprinting, Gottlieb (1961) stated that "developmental age is more sensitive than posthatch age as a baseline for the determination of the critical period in imprinting [p. 427]." In his comprehensive review of the research concerning imprinting, Sluckin (1965) concludes a description of Gottlieb's experiment by stating: "It would appear that the critical period for imprinting might be more sharply defined in terms of developmental age than in terms of conventional age [p. 77]." Similar statements, based upon Gottlieb's work, are made in several of the major textbooks in the area of animal behavior (e.g., Hinde, 1970; Marler \& Hamilton, 1966). Developmental age, age measured from the beginning of incubation, was suggested as a better baseline because various factors may cause birds to hatch at slightly different stages of maturational development. In view of the widespread acceptance and dissemination of Gottlieb's idea, it is essential that it have a firm empirical foundation. Upon reexamination of Gottlieb's data, the foundation appears to be quite flimsy.

To insure equivalent developmental stages at the beginning of incubation, Gottlieb refrigerated eggs for a period of time prior to the beginning of incubation. At various ages after hatching, he exposed 65 Peking ducklings to a male mallard decoy for $20 \mathrm{~min}$ in a typical circular runway. At 27 to $30 \mathrm{~h}$ after hatching, each $\mathrm{S}$ was exposed simultaneously to the male decoy and to one painted with female coloration. The amount of time spent following each decoy was recorded, and those Ss which followed the male decoy longer were considered to have been imprinted. Results of this testing session are presented in Fig. 1. (The top panels illustrate the data as presented in two figures by Gottlieb; the lower panels present the same data converted into percentages to facilitate comparisons.) Analysis of the data consisted of demonstrating that there were no significant differences in the periods? Obviously, it is not.

Klopfer \& Gottlieb (1962) conducted an experiment which could have clarified the situation but, unfortunately, did not. It was primarily a study of visual and auditory imprinting, but data were presented in terms of both developmental and posthatch ages. Three groups differing in posthatch age, but all between 10 and $20 \mathrm{~h}$, did not differ. Once more, analysis of developmental age was in terms of days. Data were presented for developmental age broken into 4-h intervals, but these data were not analyzed statistically. The authors did suggest, however, that " 8 to $23 \mathrm{hr}$. was the most critical period during day 27 [p. 129]." Incidentally, these data and this conclusion did not really apply to imprinting. They were based upon whether the $S$ followed during the training session; there is an indication in Gottlieb's 1961 article that following during training does not necessarily correspond with imprinting.

Although the concept of this context, and quite possibly in others, acceptance of Gottlieb's concept of usefulness should be based upon meaningful data. Perhaps Gottlieb's failure to find a clearly defined critical period, measured in terms of posthatch age, resulted from a technique for measuring imprinting which lacked sensitivity rather than from the lack of a meaningful, throughout four consecutive 5-h developmental age might be useful in

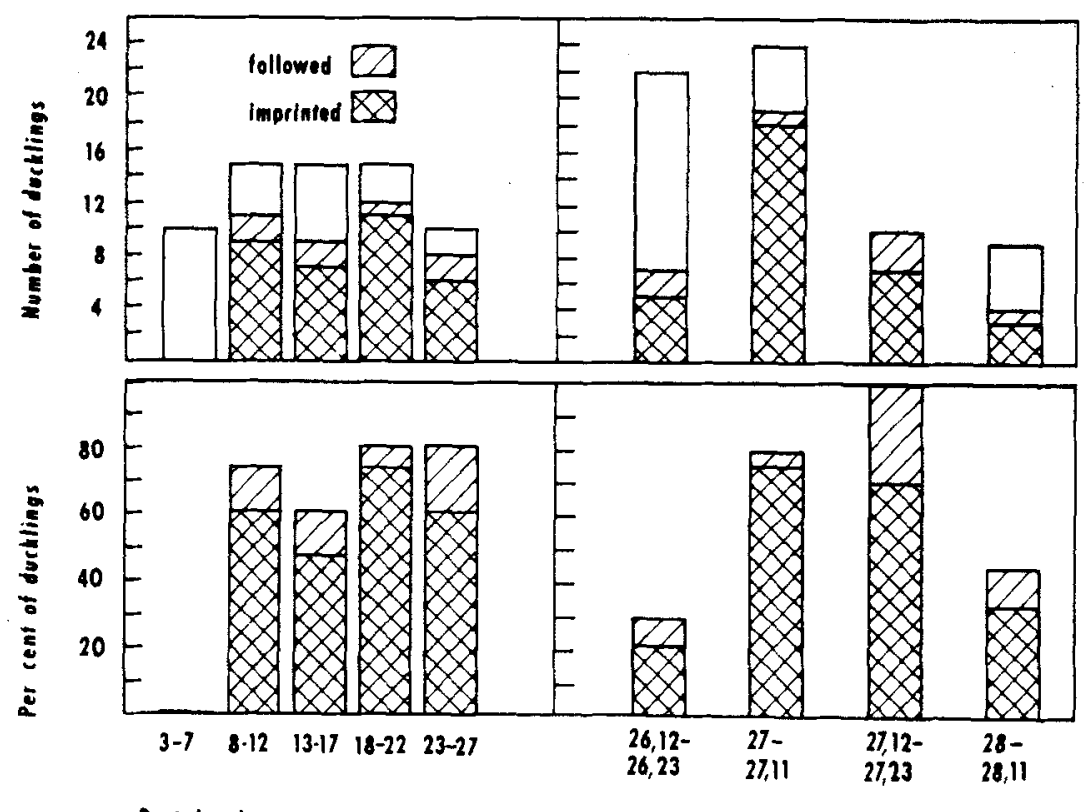

Post-hatch age of troining (hours)

Age from onsel of Incubalion (days 4 hrs)

Fig. 1. Following and imprinting as a function of posthatch age and developmental age (from Gottlieb, 1961). 
Table 1

Probability of a Minority Side Response as a Function of the Reinforcement Location on the Immediately Preceding Trial

\begin{tabular}{|c|c|c|c|c|}
\hline & \multicolumn{4}{|c|}{ Reinforcement Ratio } \\
\hline & $75: 25$ & 100:0 & $871 / 2: 121 / 2$ & $121 / 2: 87^{1 / 2}$ \\
\hline $\begin{array}{l}\text { Percent of response to.minority } \\
\text { side regardless of side reinforced } \\
\text { on the preceding trial }\end{array}$ & 10.8 & 3.7 & 8.0 & 17.4 \\
\hline $\begin{array}{l}\text { Percent of responses to minority } \\
\text { side on trials preceded by } \\
\text { majority side reinforcement }\end{array}$ & 7.9 & 3.7 & 5.6 & 11.2 \\
\hline $\begin{array}{l}\text { Percent of responses to minority } \\
\text { side on trials preceded by } \\
\text { minority side reinforcement }\end{array}$ & 23.8 & - & 25.0 & 60.8 \\
\hline $\begin{array}{l}x^{2} \text { of difference between percent } \\
\text { responses to minority side with } \\
\text { majority vs minority reinforce- } \\
\text { ment on preceding trial }(\mathrm{d} f=1 \text { ) }\end{array}$ & $\begin{array}{c}9.48 \\
p<.01\end{array}$ & - & $\begin{array}{c}14.52 \\
p<. \bar{u} \bar{i}\end{array}$ & $\begin{array}{c}53.23 \\
p<.001\end{array}$ \\
\hline
\end{tabular}

\section{RESULTS AND DISCUSSION}

The performance during the various reinforcement schedules is presented in Fig. 1. The Ss were generally quite sensitive to changes in reinforcement ratios, but they did not tend to match ratios exactly. Typical performance was intermediate between matching and maximizing. On the original problem, the performance did differ significantly from the $75: 25$ ratio $\left(\mathrm{x}^{2}\right.$ $=10.76, \mathrm{df}=1, \mathrm{p}<.01$ ). (For all statistical comparisons, data from the last 6 days of a given schedule were used.) The compromise between matching and maximizing resulted from a tendency to go to the minority side fairly often on trials which had been preceded by a reinforcement to the minority side. The probability of a response to the minority side as a function of whether the reinforcement was delivered on the majority or minority side on the immediately preceding trial is presented in Table 1 . With each ratio, there was a significantly greater tendency to go to the minority side on a trial preceded by a reinforcement on the minority side. This was particularly evident during the $12.5: 87.5$ ratio schedule after the original majority side had become the minority side. These expected residual effects from the earlier training were important, as indicated by the fact that there were significantly more responses to the minority side on the $12.5: 87.5$ ratio schedule than on the $87.5: 12.5$ ratio schedule which had preceded it $\left(x^{2}=\right.$ $3.99, \mathrm{df}=1, \mathrm{p}<.05$ ). (This difference would have been expected to disappear with extended training after the majority side was reversed.)

If one categorizes behavior during a probability matching situation into rat-like or fish-like behavior, as does Bitterman, caimans are most definitely rat-like. Their deviations from maximizing do fit the pattern of reward following, which Bitterman describes as fairly common in rats and which has been observed in some other reptiles in a spatial, but not a visual, probability matching situation (Kirk \& Bitterman, 1965). Based upon a series of studies by Mackintosh and others, Sutherland \& Mackintosh (1971) have pointed out that the term "maximizing" is generally used with reference to any asymptotic performance which exceeds the percentage of majority side reinforcement, even though it may fall short of $100 \%$. According to these authors, matching and maximizing do not represent distinct behavioral categories, but rather two points on a continuum of increasing efficiency. Level of asymptotic performance seems to be influenced by a variety of factors, which are still not completely understood, but birds are apparently more efficient than fish (reach a higher asymptote of majority choice), and rats appear to be more efficient than birds. Viewed from this standpoint, the performance of caimans on the original 75:25 problem approaches that typically demonstrated by rats. Unfortunately, as in other areas of research in comparative psychology, a vast amount of additional data representing many more species is necessary before the issues involving behavioral correlates of evolutionary development can be resolved.

\section{REFERENCES}

BITTERMAN, M. E. Phyletic differences in learning. American Psvchologist, 1965. 20,396-410.

KIRK, K. L., \& BITTERMAN, M. E Probability-learning by the turtle. Science, $1965,148,1484-1485$

SUTHERLAND, N. S., \& MACKINTOSH, N. J. Mechanisms of animal discrimination learning. New York: Academic Press. 1971.

WILLIAMS, J. T.. JR. Reversal-learning in the spectacled caiman. American Journal of Psychology, 1968, 81, 258-261.

WILLIAMS, J. T.. JR.. \& SCOTT, M. H. Reversal learning in an escape situation. Psychonomic Science, 1968, 11, 177-178. 